\title{
Asmus Finzen wird 80!
}

\section{Asmus Finzen Turns 80!}

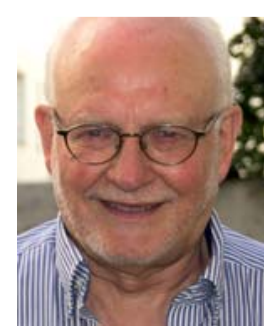

Prof. Dr. Asmus Finzen

\author{
Autor \\ Peter Brieger \\ Institut \\ kbo-Isar-Amper-Klinikum gGmbH, Akademisches \\ Lehrkrankenhaus der LMU München \\ Bibliografie \\ DOI https://doi.org/10.1055/a-1076-9801 \\ Psychiat Prax 2020; 47: 5-6 \\ (c) Georg Thieme Verlag KG Stuttgart · New York \\ ISSN 0303-4259
}

Korrespondenzadresse

Prof. Dr. Peter Brieger, Ärztlicher Direktor, kbo-Isar-Amper-

Klinikum gGmbH, Akademisches Lehrkrankenhaus der LMU

München, Vockestraße 72, 85540 Haar bei München

peter.brieger@kbo.de
Asmus Finzen hat als Psychiater und Soziologe eine so große Zahl an Projekten, Konzepten, Wahrheiten und Erfahrungen in unseren fachlichen und gesellschaftlichen Diskurs getragen, dass wir heute, kurz vor seinem 80. Geburtstag staunend vor dieser Vielfalt stehen. So hat er 1974 mit H. K. Rose und H. Köster diese Zeitschrift gegründet. Im Gründungseditorial stand damals: „Diejenigen, die in Krankenhäusern, Gesundheitsämtern und Praxen psychiatrisches Tagwerk leisten, stehen begreiflicherweise Problemlösungsangeboten zurückhaltend gegenüber, die an den konkreten Nöten vorbeientwickelt scheinen. Die Entwürfe der Sozialpsychiatrie, die Theoreme der Antipsychiatrie, die Heilslehren psychiatrischer Kollektive (...) haben den Alltag nur wenig verändert. Der inflationäre Gebrauch des Begriffs ,sozial' weckt Mißtrauen und Widerstände (...) [1].

Asmus Finzen wurde am 24.2.1940 in Taarstedt an der Ostsee geboren, er machte Abitur an der Domschule in Schleswig, studierte Soziologie und Medizin. 1965 absolvierte er sein Staatsexamen, 1968 wurde er promoviert. Es folgte 1972 die Habilitation in Tübingen - da war er gerade 32 Jahre alt, offenkundig bestand damals schon eine Frühbegabung. Finzen sagt über seine Tübinger Zeit: „Damals schien irgendwie alles auf mich zuzufliegen “ [2]. Wie manche andere fortschrittliche Psychiater ging Asmus Finzen in den späten 60er-Jahren ans Institute of Psychiatry nach London. Er besuchte dort u.a. Douglas Bennett und John Wing. In seinen Erinnerungen finden sich wunderbare Anekdoten: Beispielsweise sein Treffen mit Ronald Laing in London, den er 1968 erst einmal nicht finden konnte: „Also nahmen wir uns das Telefonbuch vor und fanden einen Dr. David Cooper in der Harley Street, einen Dr. Ronald Laing in der Upper Wimpole Street, ausgerechnet in jenen beiden Straßen, in denen sich die Londoner Ärzte mit lukrativen Privatpraxen vorzugsweise niederlassen." Es folgt eine anrührende Schilderung dieses Besuchs - mit all seinen Ambivalenzen und Widersprüchen - und schließlich eine skeptische Beobachtung: „... die Wendung, ,Leute, die andere Leute schizophren nennen“ kehrte im Gespräch mit ihm allzu oft wieder. Er versuchte dem Dilemma, Kranke etikettieren zu müssen, dadurch auszuweichen, daß er einen Purzelbaum schlug. Das Etikett war nur länger geworden“ [3].

In den 70er-Jahren war Asmus Finzen mit enormer Energie in verschiedenen Institutionen tätig: Er gehörte zu den Mitgründern des Mannheimer Kreises, der Deutschen Gesellschaft für Sozialpsychiatrie (DGSP) und der Aktion Psychisch Kranke e.V. (APK). Er war von 1971 bis 1975 Teil der Arbeitsgruppe „Intramurale Psychiatrie“ der Psychiatrie-Enquete. 1977 gründete er mit Hilde Schädle-Deininger, Klaus Dörner und Ursula Plog den Psychiatrie Verlag.

Nachhaltig beförderte er die Idee der psychiatrischen Tagesklinik - er gründete 1972 eine der ersten in Deutschland in Tübingen und leitete diese, bis er dann - wohl nicht ganz im wechselseitigen Einverständnis - die Universitätsklinik Tübingen verließ und von 1975 bis 1987 Direktor des Niedersächsischen Landeskrankenhauses Wunstorf bei Hannover wurde.

Die Leitung eines Landeskrankenhauses war ab Mitte der 70er-Jahre für manchen Reformpsychiater der Versuch, eine „bessere psychiatrische Versorgung“ zu gestalten. Das war angesichts der damaligen Verhältnisse „in den Anstalten“ eine enorme Herausforderung und ein weitreichender Auftrag - an- 
dere, die ähnliche Schritte gegangen sind, z. B. Michael von Cranach, Klaus Dörner, Nils Pörksen, Ralf Seidel oder Maria RaveSchwank, sie alle verließen die Universitäten, um aktiv daran mitzuwirken, die Versorgung neu zu gestalten. Der Weg zurück dagegen war außergewöhnlich: Asmus Finzen ist ihn 1987 gegangen und wurde Stellvertretender Ärztlicher Direktor, Leiter Allgemein- und Sozialpsychiatrie an der Psychiatrischen Universitätsklinik Basel. Diese Position hatte er bis 2003 inne, als er in den Ruhestand ging.

Es ist ein offenes Geheimnis, dass es immer wieder zu Konflikten kam, deren Spuren sich bis heute verfolgen lassen: Asmus Finzen hatte und hat Ecken und Kanten - und er hat provoziert, etwa mit seiner Streitschrift gegen die „neue Einfachheit". Schon allein der Titel war eine Provokation und wird ihm bis heute von manchen nicht verziehen: „Die neue Einfachheit oder die Entprofessionalisierung der Psychiatrie. Gegen den moralischen Pietismus in der DGSP“ [4].

Ich selbst habe Asmus Finzen erstmalig 1993 - im leuchtend orangenen Hemd und natürlich ohne Krawatte - in der eher konservativen Universitätsklinik für Psychiatrie in Frankfurt gehört, wo er kompetent und mit einer deutlich spürbaren, zutiefst menschlichen Grundhaltung einen eindrucksvollen Vortrag zum Thema Suizid gehalten hat. Er war damals ein kleiner Star unter den psychiatrischen Referenten - lebendig im Vortrag, äußerst belesen und glasklar in den Formulierungen. Diese Kompetenz, sicherlich auch seiner soziologischen Ausbildung geschuldet, hat auch dazu geführt, dass Asmus Finzen über Jahrzehnte wissenschaftsjournalistisch für die FAZ tätig war. In fast vier Jahrzehnten hat er unzählige Artikel für die FAZ zum Thema Psychiatrie verfasst. Damit hat er den gesellschaftlichen Diskurs zur Psychiatrie in der Bundesrepublik wesentlich mitgeprägt.

Er hat viel und sehr Unterschiedliches geschrieben: Sein Leitfaden zur Medikamentenbehandlung wurde erstmalig 1979 aufgelegt und erfuhr 14 Auflagen, bevor es 2017 gründlich überarbeitet erneut erschien [5]. Das Buch war viele Jahre ein Geheimtipp als Alternative zu anderen Kompendien - praxisorientierter und leichter lesbar. Andere zentrale Themen, mit denen sich Finzen befasste, sind Stigma [6, 7], Suizid und Suizidprävention - insbesondere sein Buch zum Patientensuizid ist bis heute ein Klassiker [8], aber auch mit der Sterbehilfe hat er sich differenziert befasst [9]. Hinzu kommen Tageskliniken („als Lebensschule“) [10], die NS-Krankenmorde [11] oder unfreiwillige Behandlung [12] - Themen, mit denen er oft seiner Zeit weit voraus war.

Daneben gibt es eine reichhaltige Palette von Büchern und Schriften zu Grundthemen der Sozialpsychiatrie, wie etwa der von ihm und Ulrike Hoffmann-Richter herausgegebene Band „Was ist Sozialpsychiatrie?“ [13], das „Pinelsche Pendel - Die Dimension des Sozialen im Zeitalter der biologischen Psychiatrie“ [3] und manches andere (vgl. z. B. [13, 14]). Zuletzt veröffentlichte er kluge Gedanken zur Normalität [15]. Asmus Finzen kann wunderbar schreiben, die Bücher sind schön zu lesen und gut fundiert. Sie weisen auf neue Horizonte hin.

Dann gibt es noch die Angehörigen: Asmus Finzen ist viele Jahre durchs Land gezogen und hat auf Angehörigentreffen referiert. Er hat ein Ohr und eine Sprache gefunden für ihre Nöte und Bedürfnisse, „zu zeigen, was es für eine Familie bedeutet, wenn eines ihrer Mitglieder krank wird“ [16]. Entschieden hat er sich hinter die Betroffenen und Angehörigen gestellt, Mut gemacht, Akzente gesetzt, beraten, Hinweise gegeben. Er hat in diesem Kontext auch immer wieder darauf hingewiesen, wie wichtig Sprache ist: „Der Verwaltungsrat ist schizophren“ ist für ihn ein Beispiel dafür, wie Stigmatisierung stattfindet [17].

Heute lebt Asmus Finzen in Berlin, tritt bei verschiedenen Veranstaltungen auf, diskutiert, geht abends mit einen Wein trinken, ist ein inspirierender Gesprächspartner und kritischer Geist. Er hat immer wieder provoziert und auch mal irritiert. All dies hat dazu geführt, dass er viel bewegt hat: Die Spuren, die Asmus Finzen gelegt hat und die bis heute wirken - Psychiatrische Praxis, Aktion Psychisch Kranke, Psychiatrie-Enquete, Angehörigenbewegung, Stigma, Suizidologie, Tagesklinik, Pressearbeit in der FAZ und vieles mehr - sind von einer solchen Bedeutung, dass ich, der ich um einiges jünger bin, dies mit großem Respekt sehe. Uns allen und Dir, lieber Asmus, wünsche ich zum 80., dass Du noch viele Jahre so belebend, klug und streitbar bleibst. So freue ich mich auf unsere nächsten Treffen, auf manches Neue - und über all das, was längst zum klassischen Kanon der deutschen Sozial- und Gemeindepsychiatrie gehört.

\section{Literatur}

[1] Rose H, Köster H, Finzen A. Editorial - Zielsetzung. Psychiat Prax 1974; 1: 1-3

[2] Finzen A. Erlebte Psychiatriegeschichte. Band II. Bewegte Jahre (1970 bis 1974). finzen.de/pdf-dateien/erlebte\%20psychiatriegeschichte\% 2002.pdf [8.12.2019]

[3] Finzen A. Das Pinelsche Pendel. Die Dimension des Sozialen im Zeitalter der biologischen Psychiatrie. Bonn: Edition Das Narrenschiff; 1998

[4] Finzen A. Die neue Einfachheit oder die Entprofessionalisierung der Psychiatrie. Sozialpsychiatrische Information 1981; 11: 5-20

[5] Finzen A, Scherk H, Weinmann S. Medikamentenbehandlung bei psychischen Störungen. 1. Auflage der Neuausgabe. Köln: Psychiatrie Verlag; 2017

[6] Finzen A. Stigma psychische Krankheit. Zum Umgang mit Vorurteilen, Schuldzuweisungen und Diskriminierungen. Köln: Psychiatrie Verlag; 2013

[7] Finzen A. Stigma, Stigmabewältigung, Entstigmatisierung. Psychiat Prax 2000; 27: 316-320

[8] Finzen A. Der Patientensuizid. Bonn: Psychiatrie-Verl.; 1988

[9] Finzen A. Das Sterben der Anderen. Köln: Balance buch+medien Verlag; 2009

[10] Finzen A. Die Tagesklinik. München: Piper; 1977

[11] Finzen A. Massenmord ohne Schuldgefühl. Veränderte Aufl. Bonn: Psychiatrie-Verl.; 1996

[12] Finzen A. Hilfe wider Willen. Bonn: Psychiatrie-Verl.; 1993

[13] Finzen A. Psychiatrische Praxis: Das dreißigste Jahr. Psychiat Prax 2003; 30: 413-413

[14] Wissenschaft für den psychiatrischen Alltag. Psychiat Prax 2008; 35: 2-4

[15] Finzen A. Normalität. Köln: Psychiatrie Verlag; 2018

[16] Finzen A. Schizophrenie. Die Krankheit verstehen, behandeln, bewältigen. 3. Auflage. Köln: Psychiatrie Verlag; 2020

[17] Finzen A, Benz D, Hoffmann-Richter U. Die Schizophrenie im „Spiegel“ - oder ist der Krankheitsbegriff der Schizophrenie noch zu halten? Psychiat Prax 2001; 28: 365-367 